\title{
Avaliação dos métodos farmacológicos no Transtorno do Espectro Autista (TEA): a importância da medicação no tratamento em crianças e adolescentes
}

\author{
Evaluation of pharmacological methods in Autistic Spectrum Disorder (ASD): the importance of \\ medication in the treatment of children and adolescents \\ Evaluación de métodos farmacológicos en el Trastorno del Espectro Autista (TEA): la importancia \\ de los medicamentos en el tratamiento de niños y adolescentes
}

Recebido: 28/10/2021 | Revisado: 05/11/2021 | Aceito: 08/11/2021 | Publicado: 12/11/2021

\author{
Geovanna Freitas da Rocha Nascimento \\ ORCID: https://orcid.org/0000-0002-1913-2703 \\ Centro Universitário Vale do Ipojuca, Brasil \\ E-mail: gegeofrn_11@hotmail.com \\ Paula Eduarda Marinho da Silva \\ ORCID: https://orcid.org/0000-0002-5577-5837 \\ Centro Universitário Vale do Ipojuca, Brasil \\ E-mail: paulinhaduda17@hotmail.com \\ João Paulo de Melo Guedes \\ ORCID: https://orcid.org/0000-0003-2619-5080 \\ Centro Universitário Vale do Ipojuca, Brasil \\ E-mail: joao.guedes@professores.unifavip.edu.br
}

\begin{abstract}
Resumo
O Transtorno do Espectro Autista é um transtorno do neurodesenvolvimento, que começa a se apresentar antes dos três anos de idade, sendo também classificado como uma síndrome comportamental que dá origem a uma grande variedade de sintomas clínicos. O autismo não é uma doença única, mas um transtorno complexo do desenvolvimento, definido sob a perspectiva do comportamento, com múltiplas causas e variados graus de gravidade. A medicação é uma opção para reduzir um grande número de maus comportamentos. Quando os sintomas centrais incapacitam o desenvolvimento do indivíduo e previnem os efeitos de outras terapias como por exemplo a educação e terapia comportamental, medicamentos para sintomas específicos devem ser usados. Nas últimas décadas, a prevalência de pessoas com diagnóstico de autismo aumentou, indicando a necessidade de políticas públicas que atendam às necessidades dos pacientes e seus familiares. Neste contexto, o farmacêutico desempenha um papel importante, principalmente nas orientações relacionadas aos medicamentos utilizados no tratamento.
\end{abstract}

Palavras-chave: Autismo; Tratamento farmacológico em autistas; Assistência farmacêutica; Psicofarmacologia; Medicamentos psicotrópicos e antipsicóticos.

\begin{abstract}
Autistic Spectrum Disorder is a neurodevelopmental disorder that begins to present itself before the age of three. It is also classified as behavioral syndrome that gives rise to a wide variety of clinical symptoms. Autism is not a single disease, but a complex developmental disorder, defined from a behavioral perspective, with multiple causes and varying degrees of severity. Medication is an option to reduce a large number of bad behaviors. When core symptoms cripple the individual's development and prevent the effects of other therapies such as education and behavioral therapy, medications for specific symptoms should be used. In recent decades, the prevalence of people diagnosed with autism has increased, indicating the need for public policies that meet the needs of patients and their families. In this context, the pharmacist plays an important role, mainly in the guidelines related to the drugs used in the treatment.
\end{abstract}

Keywords: Autism; Pharmacological treatment in autistics; Pharmaceutical care; Psychopharmacology; Psychotropic and antipsychotic medicines.

\section{Resumen}

El trastorno del espectro autista es un trastorno del neurodesarrollo que comienza a presentarse antes de los tres años, y también se clasifica como un síndrome conductual que da lugar a una amplia variedad de síntomas clínicos. El autismo no es una enfermedad única, sino un trastorno complejo del desarrollo, definido desde una perspectiva conductual, con múltiples causas y diversos grados de gravedad. La medicación es una opción para reducir una gran cantidad de malos comportamientos. Cuando los síntomas centrales paralizan el desarrollo del individuo y previenen los efectos de otras terapias como la educación y la terapia conductual, se deben usar medicamentos para síntomas específicos. En las últimas décadas ha aumentado la prevalencia de personas diagnosticadas con autismo, lo que indica la necesidad de 
políticas públicas que atiendan las necesidades de los pacientes y sus familias. En este contexto, el farmacéutico juega un papel importante, principalmente en las pautas relacionadas con los fármacos utilizados en el tratamiento.

Palabras clave: Autismo; Tratamiento farmacológico en autistas; Cuidado farmacéutico; Psicofarmacología; Medicamentos psicotrópicos y antipsicóticos.

\section{Introdução}

Por volta de 1911, o psiquiatra suíço Eugen Breuler introduziu o termo " autismo" na medicina. Breuler era famoso por suas pesquisas sobre esquizofrenia e classificou as pessoas que possuem dificuldade de interação social e comunicação e definiu o autismo como uma desconexão total ou parcial, das pessoas com a realidade (Pererira, 2000). Sendo que em 1943, o médico Dr. Kanner foi quem caracterizou o autismo pelo comprometimento da fala, comportamentos repetidos, contato afetivo diminuído e a dificuldade de socialização (Stelzer, 2010).

Logo depois que o autismo foi reconhecido, como uma doença diferente das outras psicoses, em 1980 ele foi introduzido como Diagnóstico Estatístico de Transtornos Mentais III - DSM-III (Cabral \& Nick, 2006; Ferreira, 2008).

A palavra autismo tem origem grega (autós) e significa: por si mesmo. Na psiquiatria usam o nome autismo para descrever um comportamento voltado para si próprio (Mello, 2007). O autismo não se classifica como uma doença única, mas sim como um transtorno complexo do desenvolvimento, definido sob a perspectiva do comportamento, com múltiplas causas e variados graus de gravidade (Rutter, 2011).

Estima-se que a prevalência atual de TEA no mundo seja de cerca de 70 casos por 10.000 habitantes e a taxa de incidência em meninos seja quatro vezes maior (Volkmar, 2014). Nos tempos atuais, ele é definido como Transtorno do Espectro Autista (TEA), determinado por nove obstáculos no desenvolvimento de várias áreas, como a comunicação e interação social. A diversidade clínica e patogenética em que se apresenta levou a definição do TEA como um grupo de distúrbios do desenvolvimento neurológico com início cedo na vida, tornando-se o autismo como uma característica comum, sendo causada por processos separados. O primeiro relato de autismo no Brasil, foi em 1987 (Bosa, 2000; Lacivita, 2017; Who, 2019).

Estudos têm demonstrado que os tratamentos com intervenções medicamentosas são utilizados como uma terapia adjuvante no transtorno do espectro autista, já que os tratamentos principais incluem intervenções psicossociais e educacionais. Dentre as classes medicamentosas mais prescritas, estão os antipsicóticos, em seguida os antidepressivos, e os anticonvulsivantes e estimulantes (Mandell et al., 2008; Arima, 2009; Brentani et al., 2013).

Contudo, com princípios em várias revisões, sabe-se que a farmacoterapia não irá tratar o transtorno do espectro autista em si, mas irá contribuir com a melhora em sintomas-alvo que compromete o comportamento dessas crianças e adolescentes, como por exemplo, a hiperatividade, insônia, agressividade e entre outros (Masi et al., 2017; Mandell et al., 2008; Brentani et al., 2013; Eissa et al., 2018).

Sendo assim, observamos a importância de levarmos as informações a toda população e profissionais da saúde sobre a doença e suas características, tendo como intuito de observar os sinais como agressividade, hiperatividade e o déficit de atenção em crianças e adolescentes portadores do TEA, destacando assim a importância do profissional farmacêutico na equipe multiprofissional que envolve o paciente.

\section{Metodologia}

Trata-se de um estudo bibliográfico do tipo narrativo no qual foram analisadas diversas publicações como livros e artigos científicos por meio eletrônico. Este estudo foi conduzido considerando o que foi apresentado por Vosgerau \& Romanowsk (2014), em que apontam que uma pesquisa de revisão narrativa, em que não há critérios metodológicos rigorosos que permitam a replicação da pesquisa e que possibilita uma visão geral do tema pautada em uma análise ampla do que se tem na literatura. Diante disso, o estudo buscou uma compreensão sobre o transtorno do espectro autista e a importância do 
farmacêutico no cuidado de crianças e adolescentes diagnosticadas com o TEA.

As pesquisas realizadas trouxeram o levantamento bibliográfico por meio das bases de dados de livros e informações encontradas em artigos científicos nas seguintes plataformas: Scientific Electronic Library Online (Scielo), Google Acadêmico e PubMed. As principais palavras chaves utilizadas nas pesquisas foram: "Tratamento farmacológico em Autistas", "Assistência Farmacêutica", "Autismo", "Psicofarmacologia", "Medicamentos Psicotrópicos e Antipsicóticos". A coleta ocorreu de forma qualitativa no qual apresenta-se pela ausência de números e estatísticas, analisando aspectos mais profundos e detalhados sobre o assunto relacionado (Dias, 2000).

Para os critérios de inclusão foram considerados artigos que apresentassem uma descrição da doença, diagnóstico, sintomas e a importância do uso da medicação correta com o intuito de ocorrer a diminuição de sinais e sintomas que prejudiquem significativamente a vida social de crianças e adolescentes com o Transtorno do Espectro Autista. Para os critérios de exclusão foi considerado quaisquer inconsistências ou irrelevâncias nos textos que fugiam do objetivo do estudo sendo assim descartadas. Foram incluídos artigos que tinham informações mais atuais para um desenvolvimento mais assertivo do estudo, e todos estavam disponíveis em inglês e português.

\section{Resultados e Discussão}

\section{Transtorno do Espectro Autista}

No ano de 1906 o psiquiatra Plouller, fez uma análise em crianças com demência infantil e foi citado por ele, pela primeira vez, o termo autismo. Mas foi em 1911, que o psiquiatra suíço Eugen Breuler introduziu o termo autismo na medicina e definiu o termo como "perda de contato com o mundo real, ocasionada pela incapacidade ou alta dificuldade de comunicação social' (Kenyon \& Miguel, 2002, p. 11). Em meados de 1943 o psiquiatra austríaco Dr. Kanner realizou um estudo com crianças autistas na faixa etária de dois aos oito anos e observou que essas crianças não tinham interesse em ter relações sociais, apresentavam comportamentos repetitivos e modificações na comunicação. (Kanner, 1943). No ano de 1944, sem saber sobre o estudo do Dr. Kanner sobre as crianças autistas, no qual só foi publicado no ano anterior outro médico psiquiatra pediatra austríaco, o Dr. Hans Asperger observou-se quatro crianças que tinham dificuldade em interação social, e descrevê-las com a condição descrita por ele de "psicopatia autística", apontando como um transtorno estável de personalidade definido pelo afastamento social (Klin, 2006).

No início dos anos 60 do século passado, os casos de autismo foram aumentando, com isso formou-se uma confusão sobre a real natureza do autismo, e algumas pessoas começaram fazer suas próprias sugestões de que o autismo poderia ser um transtorno cerebral desenvolvido desde de barriga da mãe, e podendo ser encontrado crianças com autismo em qualquer país. Foi então no ano de 1978, que o psiquiatra Michael Rutter conceituou e definiu o autismo baseado em quatro aspectos: 1) atraso e desvio sociais não só como função de retardo mental; 2) problemas de comunicação, novamente, não só em função de retardo mental associado; 3) comportamentos incomuns, tais como movimentos estereotipados e maneirismos; e 4) início antes dos trinta meses de idade. Com essa definição feita por Rutter, e algumas pesquisas a mais feitas sobre o autismo, no ano de 1980, pela primeira vez o autismo foi reconhecido e classificado no DSM-III (Diagnostic and Statistical Manual of Mental Disorders), como "transtorno invasivo do desenvolvimento" (TIDs). Esse novo termo relacionado ao autismo, conseguiu ganhar reconhecimento e acabou sendo incluído na Classificação Estatística Internacional de Doenças e Problemas Relacionados à Saúde (CID-10) (Klin, 2006, p. 2).

A classificação do TEA pode ser realizada de acordo com o QI (quociente de inteligência) da criança, sendo definido como leve, moderado e severo. A criança autista que tem o QI entre 67 e 52, se encaixa no nível leve, onde apresentam desenvolvimento considerado igual a de crianças de sua idade e conseguem ter uma vida normalmente. As crianças que têm o QI entre 51 e 36, são encaixados no nível moderado, onde conseguem ter comportamentos como execução de cuidado pessoal, 
como se vestir e se alimentar sozinhos. No nível severo, o QI está entre 35 e 20, nesse nível os autistas não conseguem executar suas atividades sozinhos e são dependentes de outra pessoa (Peeters, 1998; Motta, 2006; Ferreira, 2008).

O desenvolvimento de uma criança normal, desde que nasce sua interação com pessoas e ambientes, está presente, através de vozes, atenção em rostos sorridentes e outros estímulos. Quanto ao desenvolvimento de um bebê autista, eles não têm interesse em rostos e não demonstram interações (Klin, 2006).

A ausência ou pouco contato visual e a falta de compreensão de expressões faciais, são características que interferem na interação social do autista. Alguns indivíduos com o TEA apresentam essas características desde de pequenos e continuam com estas pelo resto da vida, o que dificulta ainda mais sua comunicação com outras pessoas, o que torna o autista muitas vezes com menor capacidade de fazer amizades e mantê-las (Brunoni; Mercadante; Schwartzman, 2014). Sendo assim, manter a convivência de crianças e adolescentes com autismo, com outras crianças da mesma idade, ajudaria a promover modelos de interação melhor, já que sua compreensão social é prejudicada. Manter esse convívio pode ajudar a evitar o isolamento entre os autistas, já que é uma característica bastante comum entre eles (Camargo \& Bosa, 2009).

\section{Epidemiologia}

No ano de 1966, na Inglaterra, o pesquisador Victor Lotter, realizou a primeira pesquisa epidemiológica sobre o autismo e relatou um índice de que a cada 10.000 crianças 4 eram autistas, isso em relação a população de um condado do noroeste de Londres. De lá até os dias atuais foram realizados diversos estudos epidemiológicos e relatados na literatura. Os índices de prevalência de estudos recentes, apontam índices que a cada 1.000 nascimentos cerca de 4 crianças nascem com autismo, 1 caso a cada 160 crianças (Klin, 2006, p. 2). O grande aumento na taxa de prevalência não se relaciona à questão de novos casos, mas a uma melhor definição no diagnóstico e investigação. Colocando assim o TEA como um problema de saúde pública (Klin, 2006; Lacitiva; 2017).

O Transtorno do autismo não possui diferença de raças e acomete em um nível maior crianças e adolescentes do sexo masculino, entretanto quando crianças e adolescentes do sexo feminino são diagnosticadas, são mais prejudicadas e sofrem mais com os sintomas (Abra, 2007; Bosa, 2006).

Não se sabe ao certo o porquê as meninas têm uma menor representação no autismo. Uma das virtudes é que os meninos possuam um limiar mais baixo para a disfunção cerebral do que as meninas, ou, ao contrário, de que as meninas possuam um prejuízo cerebral mais grave. Outras hipóteses foram estudadas e é incluída a possibilidade de que o autismo seja uma condição genética ligada ao cromossomo X, por isso, os torna mais vulneráveis. Atualmente os dados dessas hipóteses são limitados para possibilitar quaisquer conclusões (Klin, 2006, p. 2).

\section{Diagnóstico}

Os primeiros sinais se dão a partir dos 3 anos de vida da criança, neste período o que se estranha é o atraso no desenvolvimento em situações simples do dia-a-dia e a falta de comportamento normal esperado para crianças dessa idade, como por exemplo a interação social, a fala, comportamentos repetitivos, fixação por objetos das mesmas cores e entre outros aspectos (Osterling; Dawson; Munson, 2002).

Em torno de um terço das crianças diagnosticadas com autismo apresentam deficiência mental, sendo assim de suma importância identificar esses sinais diferentes o mais rápido possível, esse déficit é mais fácil de ser percebido até os 3 anos de vida dessa criança. A diferença entre ter o autismo associado ou não a essa deficiência intelectual (mental) é que a criança com TEA irá ter somente a parte de comunicação e interação social afetada (Charmant et al., 1997).

Um dos primeiros sinais percebidos nos estudos sobre o tema em crianças com Transtorno do Espectro do Autismo (TEA), é a dificuldade de olhar nos olhos e manter esse contato visual (Kanner, 1943; Jones et al., 2008). Sabendo-se que não 
pode ser visto como um sintoma único, a observação desse detalhe consegue ser percebida precocemente assim facilitando o fechamento para esse diagnóstico (Jones \& Klin, 2013).

Outra forma de se diagnosticar o autismo é pelo CID 10 (Classificação Estatística Internacional de Doenças e Problemas Relacionados à Saúde) que é estabelecido pela Organização Mundial de Saúde, onde o autismo é encontrado como " Transtorno Global do Desenvolvimento" caracterizado por alterações em interações sociais, movimentos repetitivos e estereotipado (Organização Mundial de Saúde, 2008). É importante salientar que quanto mais cedo for diagnosticado o TEA, mais cedo o início a tratamentos e estímulos, aumentando assim a possibilidade de resultados positivos no desenvolvimento do indivíduo (Araujo, 2019). Desse modo, ressalta-se a importância da equipe multidisciplinar no reconhecimento do perfil da criança com TEA com base em uma visão holística a fim de diagnosticar o transtorno e oferecer uma assistência de qualidade que atenda as necessidades individuais do paciente (Magalhães et al., 2021).

\section{Intervenção Farmacológica}

O indivíduo autista apresenta prejuízo em várias áreas, com isso é necessário que tenha um planejamento do tratamento que será utilizado em cada caso. De acordo com Bosa (2006), o tratamento adequado para crianças deve enfatizar o trabalho na fala, na interação social, educação especial e a família, em adolescentes deve-se enfatizar a terapia ocupacional e sexualidade.

Normalmente os tratamentos de primeira linha utilizados para crianças e adolescentes com autismo englobam intervenções psicossociais e ações educacionais, que tem como objetivos melhorar as habilidades sociais e comunicativas e acabar com comportamentos não adequados (Scahill; Nikolov; Jonker, 2006). Sabe-se que não existem tratamentos farmacológicos diretamente para o autismo, sendo utilizados medicamentos que auxiliam nos sintomas secundários do espectro autista, sendo proposto o controle ou diminuição deles (Brunoni; Mercadante;Schwartzman, 2014).

A utilização de fármacos na infância e adolescência está se tornando cada vez mais frequente e isso tem relação com a disponibilidade de diferentes tipos de medicamentos e o grande conhecimento sobre o diagnóstico (Rocha; Batista; Nunes, 2004).

Considerando-se que os tratamentos medicamentosos disponíveis para o autismo tratam os sintomas nucleares do espectro autista, o FDA (Food and Drug Administration, dos EUA) aprova apenas dois medicamentos, são eles, a risperidona e o aripiprazol, que auxiliam na diminuição dos sintomas (Scall; Nikolov; Jonker, 2006). É importante destacar que no caso do Brasil, os únicos medicamentos que são aprovados pela Agência de Vigilância Sanitária (ANVISA) são a Risperidona e a Periciazina, usados para manter o controle dos sintomas associados ao TEA (Brasil, 2012; Brasil, 2014).

Diferentes classes medicamentosas têm sido incluídas nessas intervenções farmacológicas com o intuito de conseguir um controle nos diversos sintomas que acompanham os pacientes do TEA. Dividida entre alguns tipos de medicamentos, os que estão em destaque são respectivamente, antipsicóticos atípicos como olanzapina, risperidona, clozapina, tendo sua finalidade tratar os sintomas secundários como a hiperatividade, agressividade, irritabilidade e automutilação; inibidores seletivos da recaptação de serotonina (ISRS) dado como exemplo o sertralina, fluoxetina e citalopram utilizados para tratar a ansiedade e comportamento repetitivo; antagonista opióide (naltrexona) e os psicoestimulantes como por exemplo o metilfenidato, os dois auxiliando no controle da hiperatividade (Eissa et al., 2018), e para distúrbios de sono é utilizado mediador do sistema nervoso central, como a melatonina (Nash \& Carter, 2016).

\section{Antipsicóticos atípicos}

Essa classe de medicamentos, originalmente foi desenvolvida para tratar psicoses. Mas nos últimos anos foi comprovado que é a classe mais segura e tolerada para se tratar os sintomas secundários do autismo. Esta classe de medicamentos inclui fármacos como risperidona, aripiprazol, clozapina, olanzapina e ziprasidona (Scahill; Nikolov; Jonker, 2006).

Esses fármacos auxiliam nos sintomas comportamentais como por exemplo, irritabilidade, agressividade e ansiedade 
(Stachnik \& Nunn-Thompson, 2007). Sua dosagem é capaz de produzir ação antipsicótica (Robertson; Matsumura; Fibiger, 1994). Eles apresentam uma menor possibilidade de efeitos adversos, sendo esse mais um dos motivos de ser a classe mais escolhida para tratar desses sintomas (Jesner, 2007).

O Risperidona como por exemplo, é um antipsicótico atípico usado bastante no TEA e teve sua aprovação pela FDE no ano de 2006 (Baribeau \& Anagnostou, 2014). Como um agonista dos receptores da dopamina e serotonina, apresentam menor incidência em comparação com os antipsicóticos de primeira geração (Brasil, 2014). É utilizado na faixa etária entre 3 e 16 anos de idade (Kim, 2015). Sendo bastante benéfico em relação a sintomas comportamentais como isolamento social, transtorno de hiperatividade e irritação. Um dos seus efeitos colaterais mais observados, é o aumento significativo do peso principalmente entre 5 e os 17 anos de idade (Jesner, 2007).

Inibidores seletivos da recaptação de serotonina (ISRS)

A serotonina é responsável pela regulação do sono, percepção de dores,apetite e entre outros. Sua síntese é realizada a partir de um aminoácido chamado de triptofano, dentro da célula nervosa (Silva \& Andrade, 2008). Os principais medicamentos utilizados foram os antidepressivos tricíclicos que são importantes inibidores da recaptação de serotonina e noradrenalina, além de ser um forte antagonista de receptores muscarínicos (Davies, 1971).

A elevação do nível de serotonina em crianças e adolescentes autistas é uma característica importante em até $25 \%$ dos pacientes (Cook, 1996). Ela reflete em vários comportamentos observados, como por exemplo a ansiedade, depressão e transtornos de sono. O medicamento atua no neurônio impedindo o transporte desse neurotransmissor, formando seu efeito principal que é o antidepressivo (Mycek, 1998).

Os comportamentos repetitivos geralmente encontrados em crianças com TEA, juntamente de obsessões e comportamentos compulsivos, é onde é usado a terapêutica com a medicação dessa classe, particularmente um dos mais utilizados é a Fluoxetina e o Citalopram (Peñagarikano, 2015). A fluoxetina trouxe benefícios mais significativos para a população adulta, principalmente na sintomatologia do transtorno compulsivo (Sung et al., 2014).

Antagonistas opióides

Após alguns estudos foi comprovado uma diminuição significativa nos comportamentos de hiperatividade e autoagressivo com a intervenção farmacológica com o naltrexona. Foi observado também, a melhora em outros sintomas característicos como a agitação e a interação social (Lee et al., 2014). Segundo Roy, Roy, Deb e Unwing (2015), alguns efeitos adversos vistos e destacados, foram respectivamente a perda de peso, em cerca de $60 \%$ dos pacientes e diminuição do apetite e sonolência numa menor quantidade.

Exemplo de uma medicação dessa classe é o Naltrexona, que possui uma biodisponibilidade por via oral e sua eliminação é de meia-vida prolongada e todos os seus efeitos duram no corpo 24 horas (Reisine \& Pasternak, 1996). Sua administração é feita por via oral e é absorvido em no máximo uma hora. A relação do Naltrexona e o autismo é na melhoria em sintomas secundários, como por exemplo atitudes violentas. Possui seu efeito de primeira passagem no fígado e cerca de $95 \%$ do fármaco é transformado em beta-naltrexona,que é seu metabólico na forma ativa (Campbell et al., 1998).

\section{Psicoestimulantes}

É a principal classe farmacológica usada no tratamento do transtorno de déficit de atenção com hiperatividade (TDAH), que possui sintomas bastantes comuns com o transtorno do espectro autista (TEA). Com isso o aumento por essa classe terapêutica aumentou cerca de cinco vezes mais entre a população com o TEA, no tempo de 2003 a 2010 (Baribeau \& Anagnostou, 2014). Os benefícios do uso a longo prazo desses medicamentos bem administrados, foi observado por um grande e recente estudo realizado pelo o National Institute for Mental Health (NIMH), (Arch, 1999).

O único psicoestimulante disponível e considerado droga de primeira escolha para o TEA no Brasil, é o 
metilfenidato(ritalina), e nos demais países o psicoestimulante usado é dextroanfetamina, que no Brasil não é permitido o seu uso (Popper, 2000). Sendo o fármaco mais utilizado na intervenção terapêutica do TEA, o metilfenidato possui efeito estimulante no sistema nervoso central, ele é conhecido por ser indicado para o tratamento de transtorno de déficit de atenção e hiperatividade (TDAH) (Eissa et al., 2018).

Um estudo realizado pela Research Units on Pediatric Psychopharmacology Autism Network (RUPPAN), avaliou as dosagens do metilfenidato administradas três vezes ao dia por pacientes com o TEA, e foi observado benefícios relativos a sintomatologia do autismo, como a hiperatividade, déficit na atenção e impulsividade (Autisn, 2005). Devido a seus benefícios na hiperatividade, o metilfenidato desperta grande interesse no seu uso em crianças e adolescentes com TEA, sendo fundamental mais estudos serem feitos para retirar mais conclusões (Baribeau \& Anagnostou, 2014). Apesar de ter vários benefícios no autismo, sugere-se que a eficácia do metilfenidato não é tão evidente em portadores do TEA quando comparada a portadores do TDAH (Sung et al., 2014).

\section{Mediador do Sistema Nervoso Central}

Segundo Nash,2016, o fármaco de interesse nessa classe é a melatonina (N-acetil-5-metoxitriptamina). A melatonina, é um neurohormona endógena, produzida e sintetizada pela glândula pineal durante a noite, sendo liberada como hormônio circulante. Ela produz uma secreção que é controlada pela onda de luz, por isso sua atividade durante o período da noite (Rang et al., 2016).

É considerado que indivíduos com o TEA, apresentam uma disfunção no funcionamento da glândula pineal, tornando assim uma deficiência nos níveis de melatonina. Como sua função é estabelecer os ritmos circadianos e desempenhar um papel na regulação da intervenção sináptica, a disfunção nessa síntese, resulta nos baixos níveis de melatonina, ocasionando assim aos distúrbios do sono (Shomrat \& Nesher, 2019).

A melatonina foi e continua sendo estudada como terapêutica no TEA, pois tem demonstrado alguns benefícios nas perturbações do sono e sintomas comportamentais, como eficácia no sono, diminuição da frequência do despertar noturno, benefícios na ansiedade dos indivíduos com TEA, nos comportamentos estereotipados e no isolamento social (Rossignol \& Frye, 2011). De acordo com Rossignol \& Frye (2011) a prevalência do uso de melatonina como terapêutica em doentes com o TEA é cerca de 7\%. Dessa maneira, o uso da melatonina para intervir nas perturbações do sono no TEA, se mostra como uma boa alternativa no tratamento com neurolépticos ou ansiolíticos, alguns estudos sugerem a eficácia da melatonina, sendo que são necessárias mais alguns estudos para que sua evidência seja totalmente eficaz (Colineaux et al., 2011).

\section{Assistência Farmacêutica}

A assistência farmacêutica é um conjunto de ações voltadas à promoção, proteção e recuperação da saúde, tanto individual como coletivo, tendo o medicamento como insumo essencial e visando o acesso ao seu uso racional. É possível dizer que a assistência farmacêutica é o campo que exerce ações para promover o acesso da população a medicamentos essenciais, aqueles no qual satisfazem as necessidades prioritárias da saúde da população (OMS, 2002).

Como se sabe o profissional farmacêutico é capacitado para garantir a qualidade dos medicamentos, e em conduzir uma orientação correta sobre o uso de medicamentos contribuindo assim para qualidade de vida dos seus pacientes (Ferreira, 2011). O exercício da assistência farmacêutica proporciona o uso racional de medicamentos, levando assim a conscientização do paciente e seus familiares em relação à necessidade e importância do uso de medicamentos (Silva; Naves; Vidal, 2008).

A orientação farmacêutica é realizada em várias etapas, no qual o profissional farmacêutico realiza uma entrevista com o paciente para que consiga identificar o problema da terapia medicamentosa, e assim junto a outros profissionais, consiga estabelecer um tratamento adequado e definir um plano terapêutico que possa fazer o acompanhamento para resolver e evitar 
problemas relacionados ao uso dos medicamentos (Oliveira et al., 2015). Em relação ao TEA, o seu tratamento envolve vários medicamentos, no qual é necessário que tenham atenção e que seja realizada uma boa administração dos mesmos, tendo em vista que a maioria dos pacientes são crianças e adolescentes. Ou seja, como os medicamentos são administrados por terceiros, que normalmente são os pais ou responsáveis, pode ocorrer de maneira errada e não levar a ter um sucesso na sua terapia (Fernandes et al.., 2017).

\section{Considerações Finais}

Tendo em vista o aumento significativo de crianças e adolescentes com o diagnóstico fechado para o autismo e sabendo que os estudos sobre o transtorno ainda são incertos, torna-se necessário aprofundar as pesquisas relacionadas ao tratamento e diagnóstico. Diante disso, sugere-se a realização de estudos randomizados de larga escala a fim de reunir evidências que possam embasar condutas profissionais visando a melhor qualidade de assistência prestada a esta população.

O TEA tem seu desenvolvimento na primeira infância, e se estende até a vida adulta, pois como se sabe sua cronicidade e variedade de sintomas é bastante extensa. A criança diagnosticada com TEA, apresenta déficits comportamentais, como por exemplo, atraso na fala, comportamentos repetitivos e restritivos e introspectividade. O seu diagnóstico deve ser precoce, existem algumas ferramentas que auxiliam nesse processo como por exemplo a observação dos pais no início de desenvolvimento da criança, para que assim contribua com um tratamento adequado.

Visto que, o papel do farmacêutico se torna importante no tratamento do paciente autista, por levar a informação aos familiares e pacientes sobre efeitos adversos e colaterais, interações de medicamentos e/ou alimentos, posologia e uso racional da medicação.

Sobre a intervenção farmacológica foi observado nas literaturas, o uso do risperidona dirigida principalmente para a sintomatologia associada. $\mathrm{O}$ aripiprazol e o risperidona (antipsicóticos atípicos) são os únicos fármacos no mundo aprovados pelo FDA para o uso. Outras classes como inibidores da recaptação da serotonina (Citalopram) usados para tratar sintomas da depressão e psicoestimulantes (Ritalina) utilizados em casos de hiperatividade e TDAH. O presente estudo conclui então que as classes farmacológicas que possuem estudos sobre o TEA auxiliam significativamente na qualidade de vida dos mesmos, sendo responsável juntamente com tratamento não farmacológico na diminuição de sintomas comportamentais.

\section{Referências}

APA. (2013). Diagnostic and statistical manual of mental disorders (DSM-5). American Psychiatric Pub, American Psychiatric Association.

ABRA. (2021). Autismo. Associação Brasileira de Autismo. www.autismo.org.br/

Araújo, J. (2004). Oxidação de lipídios em alimentos.In:Araújo, Jma Química de alimentos: teoria e prática,3, 1-64

Barros Neto, S. G. D., Brunoni, D., \& Cysneiros, R. M. (2019). Abordagem psicofarmacológica no transtorno do espectro autista: uma revisão narrativa. Cadernos de Pós-Graduação em Distúrbios do Desenvolvimento, 19(2), 38-60.

Bosa, C. A. (2006). Autismo: intervenções psicoeducacionais. Brazilian Journal of Psychiatry, 28, s47-s53.

Colineaux, C., Doyen, C., Mighiu, D., Kaye, K., Beaumanoir, C., Mouraeff, Y., \& Contejean, Y. (2011). Melatonina em crianças com transtornos do espectro autista: dados recentes e práticos. Psiquiatria infantil e adolescente europeia, 20 (5), 231-239.

Dourado, F. (2012). Autismo e Cérebro Social: compreensão e ação. Premius.

Eissa, N., Al-Houqani, M., Sadeq, A., Ojha, S. K., Sasse, A., \& Sadek, B. (2018). Esclarecimento atual sobre a etiologia e o tratamento farmacológico do transtorno do espectro do autismo. Fronteiras em neurociência, 12, 304.

Ferreira, M. J. Q. (2011). Assistência Farmacêutica Pública: uma revisão de literatura.

Kim, S. K (2015). Atualização recente dos transtornos do espectro do autismo. Jornal coreano de pediatria, 58 (1), 8. 
Lacivita, E., Perrone, R., Margari, L., \& Leopoldo, M. (2017). Alvos para terapia medicamentosa para transtorno do espectro do autismo: desafios e direções futuras. Journal of medicinal Chemistry, 60 (22), 9114-9141.

Lee, Y. J., Oh, S. H., Park, C., Hong, M., Lee, A. R., Yoo, H. J., \& Bahn, G. H (2014). Farmacoterapia avançada evidenciada pela patogênese do transtorno do espectro do autismo. Clinical Psychopharmacology and Neuroscience, 12(1):19- 30.

Magalhães, J. M., Silva, T. M. da, Silva, F. da C., Alencar, M. de F. B. de, Rêgo Neta, M. M., Alencar, D. de C., \& Arisawa, E. A. L. S. (2021). Profile of children with autism spectrum disorder. Research, Society and Development, 10(4), e3710413880.

Mesquita, W. S., \& Pegoraro, R. F. (2013). Diagnóstico e tratamento do transtorno autístico em publicações brasileiras: revisão de literatura. J Health Sci Inst, 31(3), 324-9.

Ministério da Saúde. (2014). Risperidona no Transtorno do Espectro do Autismo (TEA). Relatório de Recomendação da Comissão Nacional de Incorporação de Tecnologias no SUS - CONITEC - 123. Brasília, DF: Ministério da Saúde.

Newschaffer, C. J., Croen, L. A., Daniels, J., Giarelli, E., Grether, J. K., Levy, S. E., Mandell, D. S., Miller, L. A., Pinto-Martin, J., Reaven, J., Reynolds, A. M., Rice, C. E., Schendel, D., \& Windham, G. C. (2007). A epidemiologia do transtorno do espectro do autismo. Revisão Anual de Saúde Pública, 28, 235-258.

Nikolov, R., Jonker, J., \& Scahill, L. (2006). Autismo: tratamentos psicofarmacológicos e áreas de interesse para desenvolvimentos futuros. Brazilian Journal of Psychiatry, 28, s39-s46.

Osterling, J. A, Dawson, G., \& Munson, J. A. (2002). Reconhecimento precoce de bebês de 1 ano de idade com transtorno do espectro do autismo versus retardo mental. Desenvolvimento e psicopatologia, 14 (2), 239-251.

Seleção de Medicamentos Essenciais. (2002) In: Perspectivas Políticas sobre Medicamentos da OMS - 4. Genebra: Organização Mundial da Saúde.

Peñagarikano, O. (2015). Novas opções terapêuticas para o transtorno do espectro do autismo: evidências experimentais. Neurobiologia experimental, 24 (4), 301.

Rang H. P. et al. (2016). Farmacologia. (8a ed.) Elsevier.

Rossignol, D. A., \& Frye, R. E. (2011). Melatonin in autism spectrum disorders: a systematic review and meta-analysis. Developmental Medicine \& Child Neurology, 53(9), 783-792.

Roy, A., Roy, M., Deb, S., Unwin, G. (2015). Are opioid antagonists effective in attenuating the core symptoms of autism spectrum conditions in children: a systematic review. Journal of Intellectual Disability Research, 59(4): 783-792.

Stelzer, F. G. (2010). Uma pequena história do autismo: Cadernos de Pandorga de Autismo. Editora Oikos 38 p.

Sung, M., Chin, C. H., Lim, C. G., Liew, H. S. A., Lim, C. S., Kashala, E., \& Weng, S. J. (2014). O que está no pipeline? Drogas em desenvolvimento para o transtorno do espectro do autismo. Doença neuropsiquiátrica e tratamento, 10, 371.

Vosgerau, D. S. A. R., \& Romanowski, J. P. (2014). Estudos de revisão: implicações conceituais e metodológicas. Revista diálogo educacional, 14(41), 165-189. 